\title{
Thermally induced band gap increase and high thermoelectric figure of merit of n-type $\mathrm{PbTe}$
}

\author{
Jiang Cao ${ }^{\mathrm{a}, \mathrm{b}, *}$, José D. Querales-Flores ${ }^{\mathrm{b}}$, Stephen Fahy ${ }^{\mathrm{b}, \mathrm{c}}$, Ivana Savić ${ }^{\mathrm{b}, * *}$ \\ ${ }^{a}$ School of Electronic and Optical Engineering, Nanjing University of Science and Technology, Nanjing 210094, China \\ ${ }^{b}$ Tyndall National Institute, Dyke Parade, Cork T12 R5CP, Ireland \\ ${ }^{c}$ Department of Physics, University College Cork, College Road, Cork T12 K8AF, Ireland
}

\section{Abstract}

Unlike in many other semiconductors, the band gap of PbTe increases considerably with temperature. We compute the thermoelectric transport properties of $\mathrm{n}$-type $\mathrm{PbTe}$ from first principles including the temperature variation of the electronic band structure. The calculated temperature dependence of the thermoelectric quantities of $\mathrm{PbTe}$ is in good agreement with previous experiments when the temperature changes of the band structure are accounted for. We also calculate the optimum band gap values which would maximize the thermoelectric figure of merit of $\mathrm{n}$-type $\mathrm{PbTe}$ at various temperatures. We show that the actual gap values in $\mathrm{PbTe}$ closely follow the optimum ones between $300 \mathrm{~K}$ and $900 \mathrm{~K}$, resulting in the high figure of merit. Our results indicate that an appreciable increase of the band gap with temperature in direct narrow-gap semiconductors is very beneficial for achieving high thermoelectric performance.

Keywords: Thermoelectric transport properties, Boltzmann transport equation, first-principles, temperature-dependent band structure

\section{Introduction}

Thermoelectric (TE) materials convert reversibly thermal energy to electrical energy. The TE figure of merit is defined as $z T=\sigma S^{2} T / \kappa$, where $\sigma$ is the electrical conductivity, $S$ is the Seebeck coefficient, $T$ is the temperature, and $\kappa$ is the thermal conductivity [1]. The efficiency of TE refrigerators and power generators increases with larger values of $z T$. At higher temperatures, minority carriers caused by thermal activation contribute negatively to Seebeck coefficient and decrease $z T$ [2]. An increasing band gap with temperature can suppress these bipolar effects and increase $z T$. Such band gaps would also lead to larger effective masses and Seebeck coefficient at higher $T$ in direct gap semiconductors [3].

Previous model calculations have determined the ideal values of band gaps that would result in maximal $z T$ values for semiconductors with indirect and direct gaps and relatively parabolic bands [4, 5, 6], For direct gap semiconductors, if the band gap $E_{G}$ is smaller than $6 k_{B} T$, where $k_{B}$ is the Boltzmann constant, it was found that $z T$ decreases when $E_{G}$ decreases due to the presence of minority carriers. If $E_{G}>10 k_{B} T, z T$ may decrease or increase with increasing $E_{G}$ depending on the dominant electron scattering mechanism in the material. However, for real TE materials, where different scattering mechanisms compete, no previous work has calculated the optimum band gap values at different temperatures from first principles.

\footnotetext{
${ }^{*}$ Corresponding author

${ }^{* *}$ Corresponding author

Email addresses: jiang. cao@njust.edu.cn (Jiang Cao), ivana.savic@tyndall.ie (Ivana Savić)
}

In most semiconductors, the band gap decreases with increasing temperature. A particularly interesting exception is the group of lead chalcogenides ( $\mathrm{PbS}, \mathrm{PbSe}, \mathrm{PbTe}$ ), whose direct narrow gap increases with increasing temperature [7, 8, 9]. A positive temperature coefficient of the band gap, $\partial E_{G} / \partial T$, could lead to the optimum gap values in a wider temperature range than if this coefficient is negative. The band gap of $\mathrm{PbTe}$ increases from $0.19 \mathrm{eV}$ at $30 \mathrm{~K}$ to $0.38 \mathrm{eV}$ at $500 \mathrm{~K}$ [8, 9] $\left(\partial E_{G} / \partial T \approx 4.7 k_{B}\right)$. Recent calculations suggested that this peculiar increase of $E_{G}$ with $T$ may be correlated with large anharmonicity [10]. Our recent first-principles work revealed that this effect stems from the Debye-Waller and thermal expansion contributions to the temperature renormalization of $\mathrm{PbTe}$ 's gap [11].

First-principles calculations of thermoelectric transport quantities are typically carried out using the electronic band struc-

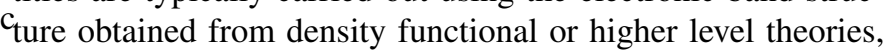
where the band shifts due to temperature are not accounted for [12, 13, 14, 15, 16, 17, 18, 19, 20]. For example, recent $a b$ initio calculations of TE transport in PbTe used a fixed band gap value at all temperatures [17]. In contrast, an earlier study indicated the importance of accounting for the temperature variations of the band structure when modelling TE transport in $\mathrm{PbTe}[21,22]$. Our previous work on this subject [23] focused on the electronic mobility of n-type $\mathrm{PbTe}$ up to room temperature, where this effect is not so prominent.

In this work, we calculate all thermoelectric transport properties of $\mathrm{PbTe}$ from first principles, explicitly accounting for the temperature renormalization of the electronic band structure. We find much better agreement between our results and 
experiments when we include the temperature induced changes of the band structure. The ideal band gap values that would maximize $z T$ at different temperatures have also been obtained. The optimum gap values vary from $10 k_{B} T$ at $300 \mathrm{~K}$ to $6.5 k_{B} T$ at $900 \mathrm{~K}$, due to the competition between longitudinal optical and acoustic phonon scattering at high doping concentrations and temperatures. The actual band gap values of $\mathrm{PbTe}$ are very similar to the optimum ones in the whole temperature range. Therefore, the sizeable positive temperature coefficient of the narrow band gap in $\mathrm{PbTe}$ enables its high $z T$ values over a large $T$ range. This work suggests that efficient thermoelectric materials with a broad working temperature range may be found among direct narrow gap semiconductors with relatively large positive values of $\partial E_{G} / \partial T$ (several $\left.k_{B}\right)$.

\section{Method}

\subsection{Temperature-dependent band structure}

$\mathrm{PbTe}$ is a direct narrow gap semiconductor with the gap located at four equivalent $\mathrm{L}$ points. The $\mathrm{L}$ valleys give the largest contribution to the electronic conduction and TE transport in n-type PbTe [23]. At higher temperatures, the energies of the valence band maxima at $\Sigma$ become similar to those of the valence band maxima at L. We neglect this effect in the present calculations. The electronic band structure near the $\mathrm{L}$ point in $\mathrm{PbTe}$ is well described using the two-band Kane model derived from $\boldsymbol{k} \cdot \boldsymbol{p}$ theory [3]. The energy dispersion in the two-band Kane model is non-parabolic, and for the band extrema at the $\mathrm{L}$ point satisfies the relation:

$$
\frac{\hbar^{2}}{2}\left(\frac{k_{\|}^{2}}{m_{\|}^{*}}+\frac{k_{\perp}^{2}}{m_{\perp}^{*}}\right)=E\left(1+\frac{E}{E_{G}}\right),
$$

where $E_{G}$ is the direct band gap, $k_{\|}$and $k_{\perp}$ are the components of the wavevector parallel and perpendicular to the $\Gamma$ - $\mathrm{L}$ direction, respectively, and $m_{\|}^{*}$ and $m_{\perp}^{*}$ are the parallel and perpendicular effective masses, respectively. In the two-band Kane model, the valence band (VB) is a mirror image of the conduction band $(\mathrm{CB})$ with the dispersion given as $\left(-E_{G}-E\right)$.

The band gap and the effective masses for the ground state of PbTe are obtained from density functional theory (DFT) calculations, using the Vienna ab-initio simulation package (VASP) We use the screened Heyd-Scuseria-Ernzerhof (HSE03) hybrid functional [25, 26] and include the spin-orbit coupling (SOC), which correctly reproduces the experimental values of the band gap and effective masses at low temperatures [27, 23]. The basis set for the one-electron wave functions is constructed with the Projector Augmented Wave (PAW) method [28]. For the PAW pseudopotentials, we include the $5 d^{10} 6 s^{2} 6 p^{2}$ states of $\mathrm{Pb}$ and $5 s^{2} 5 p^{4}$ states of Te as the valence states. A cutoff energy of $18.4 \mathrm{Ha}$ and a $8 \times 8 \times 8 \boldsymbol{k}$-mesh are used for the electronic band structure of PbTe.

The temperature induced renormalization of the electronic band gap is due to thermal expansion and electron-phonon coupling [29, 30]. We calculate the gap variation due to thermal expansion using the lattice constant values that account for thermal expansion, and computing the corresponding band gap change using DFT [11]. We compute the temperature dependence of the lattice constant using lattice dynamics from first principles, as explained in Ref. [11]. The electron-phonon renormalization of the band gap is calculated using the Allen-Heine-Cardona theory [31, 32, 33] and its density functional perturbation theory (DFPT) implementation in the ABINIT code [34, 35]. We use the local density approximation (LDA) [36, 37] and HartwigsenGoedecker-Hutter norm-conserving pseudopotentials [38] with the $6 s^{2} 6 p^{2}$ states of $\mathrm{Pb}$ and $5 s^{2} 5 p^{4}$ states of Te explicitly included in the valence states. Spin-orbit interactions are included. We use the cutoff energy of $45 \mathrm{Ha}$, and a $12 \times 12 \times 12$ MonkhorstPack $\boldsymbol{k}$-point grid.

We calculate the temperature dependence of the band gap of $\mathrm{PbTe}$ with respect to its LDA value, $\Delta E_{G}(T)=E_{G}(T)-$ $E_{G}(\mathrm{LDA})$, and its temperature derivative $\partial E_{G} / \partial T$. These temperature changes are added to the band gap values obtained using the HSE03 functional to obtain $E_{G}(T)$ used in the two-band Kane model. The effective masses of the renormalized bands near $\mathrm{L}$ at a finite temperature are then computed using the twoband Kane model as [39]

$$
m_{d}^{*}(T) / m_{d}^{*}(0 \mathrm{~K})=E_{G}(T) / E_{G}(0 \mathrm{~K}),
$$

where $d$ denotes the parallel or perpendicular direction. We note that due to the small calculated value of the zero-point renormalization of the gap $(\sim 20 \mathrm{meV})$ [11], we approximate the values of the effective masses at $0 \mathrm{~K}$ with their values obtained using the HSE03 functional.

\subsection{Thermoelectric properties}

To study thermoelectric transport in n-type PbTe, we use the Boltzmann transport theory within the relaxation time approximation. Thermoelectric transport properties can be calculated as

$$
\begin{aligned}
\sigma^{i j} & =L_{0}^{i j}, \\
S^{i j} & =-L_{1}^{i j} /\left(e T L_{0}^{i j}\right), \\
\kappa_{0}^{i j} & =L_{2}^{i j} /\left(e^{2} T\right),
\end{aligned}
$$

where $\sigma$ is the dc electrical conductivity tensor, $S$ is the Seebeck coefficient tensor, $\kappa_{0}$ is the thermal conductivity tensor de24 fined when the electric field across the material is zero, $i$ and $j$ are the Cartesian directions, and $e$ is the electron charge. The transport kernel functions for the $\mathrm{CB}$ are defined by

$$
L_{\alpha}^{i j(e)}=\int_{\mathrm{BZ}} \frac{e^{2} d \boldsymbol{k}}{4 \pi^{3}}\left(-\frac{\partial f}{\partial E}\right) \tau_{\boldsymbol{k}, \mathrm{tot}} v_{\boldsymbol{k}}^{i} v_{\boldsymbol{k}}^{j}\left(E_{\boldsymbol{k}}-E_{F}\right)^{\alpha},
$$

where $E_{\boldsymbol{k}}$ and $v_{\boldsymbol{k}}^{i}$ are the energy and the group velocity of an electronic state with the crystal momentum $\boldsymbol{k}, f$ is the equilibrium Fermi-Dirac occupation function, $E_{F}$ is the Fermi level, and $\tau_{k \text {,tot }}$ is the relaxation time. Unlike $\kappa_{0}$, the total thermal conductivity $\kappa$ is defined at zero electric current across the material, and is the sum of the lattice contribution $\kappa_{L}$ and the electronic contribution $\kappa_{e}$, which can be given as

$$
\kappa=\kappa_{L}+\kappa_{e}=\kappa_{L}+\kappa_{0}-T \sigma S^{2} .
$$


Since $\mathrm{PbTe}$ is cubic, $\sigma^{i j}, S^{i j}$, and $\kappa_{0}^{i j}$ can be expressed as

$$
\sigma^{i j}=\delta_{i j} \sigma ; \quad S^{i j}=\delta_{i j} S ; \kappa_{0}^{i j}=\delta_{i j} \kappa_{0} ;
$$

where $\delta_{i j}$ is the Kronecker delta, and $\sigma, S$, and $\kappa_{0}$ are given as: $\sigma=\sum_{i} \sigma^{i i} / 3, S=\sum_{i} S^{i i} / 3$, and $\kappa_{0}=\sum_{i} \kappa_{0}^{i i} / 3$.

We note that the superscript index $(e)$ in Eq. (4) refers to electrons. We also include the contribution from holes by treating the VB as a mirror-image of the CB in the two-band Kane model. The analogue definitions of the transport kernel functions for holes are obtained by simply substituting $E_{F}$ by $\left(-E_{G}-\right.$ $E_{F}$ ) in Eq. (4). By combining the contributions from electrons and holes, we obtain the total transport kernel functions $L_{\alpha}=L_{\alpha}^{(e)}+L_{\alpha}^{(h)}$. Our calculations show that the effect of holes on the electronic transport properties is negligible at the optimum doping concentrations for TE applications $\left(\sim 10^{19} \mathrm{~cm}^{-3}\right)$.

The total relaxation time $\tau_{k \text {,tot }}$ is determined by the contributions of different scattering mechanisms: acoustic (ac) phonons, transverse optical (TO) phonons, longitudinal optical (LO) phonons, and ionized impurities (imp) [17, 23, 40, 41], and can be calculated via Matthiessen's rule:

$$
\tau_{k, \text { tot }}^{-1}=\tau_{k, \text { ac }}^{-1}+\tau_{k, \mathrm{LO}}^{-1}+\tau_{k, \mathrm{TO}}^{-1}+\tau_{k, \mathrm{imp}}^{-1} .
$$

The relaxation time of a single scattering channel is given by [14 15]

$$
\tau_{k}^{-1}=\frac{2 \pi}{\hbar} \sum_{q} \frac{1-f_{k^{\prime}}}{1-f_{k}}\left(1-\hat{\boldsymbol{v}}_{\boldsymbol{k}} \cdot \hat{\boldsymbol{v}}_{\boldsymbol{k}^{\prime}}\right) S_{\boldsymbol{k}}^{\boldsymbol{k}^{\prime}},
$$

where $S_{\boldsymbol{k}}^{\boldsymbol{k}^{\prime}}$ denotes the transition rate from initial $\boldsymbol{k}$ to final state $\boldsymbol{k}^{\prime}$ due to scattering, and $\hat{\boldsymbol{v}}_{\boldsymbol{k}}$ is the unit vector in the direction of the group velocity at $\boldsymbol{k}$. The velocity factor $\left(1-\hat{\boldsymbol{v}}_{\boldsymbol{k}} \cdot \hat{\boldsymbol{v}}_{\boldsymbol{k}^{\prime}}\right)$ accounts for the change of direction of scattered carriers. For electron-phonon scattering, we can write

$$
\begin{aligned}
S_{k}^{k+\boldsymbol{q}}=\left|g_{\boldsymbol{k}}^{k+\boldsymbol{q}}\right|^{2} & \left\{N^{0}\left(\omega_{\boldsymbol{q}}\right) \delta\left(E_{\boldsymbol{k}}+\hbar \omega_{\boldsymbol{q}}-E_{\boldsymbol{k}+\boldsymbol{q}}\right)\right. \\
+ & {\left.\left[N^{0}\left(\omega_{\boldsymbol{q}}\right)+1\right] \delta\left(E_{\boldsymbol{k}}-\hbar \omega_{\boldsymbol{q}}-E_{\boldsymbol{k}+\boldsymbol{q}}\right)\right\}, }
\end{aligned}
$$

where $N^{0}$ and $\omega_{\boldsymbol{q}}$ are the equilibrium distribution and the frequency of a phonon with the crystal momentum $\boldsymbol{q}$, and $g_{\boldsymbol{k}}^{\boldsymbol{k}+\boldsymbol{q}}$ is the electron-phonon matrix element. The two terms in the curly brackets correspond to phonon absorption and emission, respectively.

We parametrize the electron-phonon matrix elements $g_{k}^{k+q}$ due to acoustic, TO and LO phonons as described in detail in our previous work [23]. This requires calculations of acoustic and optical deformation potentials, phonon frequencies, elastic and dielectric constants [23]. Some of these parameters, such as phonon frequencies, elastic and dielectric constants, can be computed straightforwardly from first principles, using DFPT. Our earlier work [27] describes several methods to calculate acoustic deformation potentials of PbTe from first principles, one of which uses DFPT. We use the same DFPT method to obtain optical deformation potentials of PbTe. The values of all these parameters are listed in Table I of Ref. [23]. Since the VB is described as a mirror image of the $\mathrm{CB}$, the absolute values of deformation potentials for the $\mathrm{VB}$ of $\mathrm{PbTe}$ are taken to be the same as those for the CB. All these parameters are calculated using the LDA excluding SOC that gives a positive band gap and the correct character of the conduction and valence band states near the $\mathrm{L}$ point in $\mathrm{PbTe}$, in contrast to the LDA including SOC [27] We note that we do not include the temperature changes of the parameters characterizing the strength of electron-phonon coupling in this work. We also account for ionized impurity scattering using the Brooks-Herring model with the Thomas-Fermi model for carrier screening, which was also used for screening of LO phonon scattering [23].

\section{Results and Discussion}
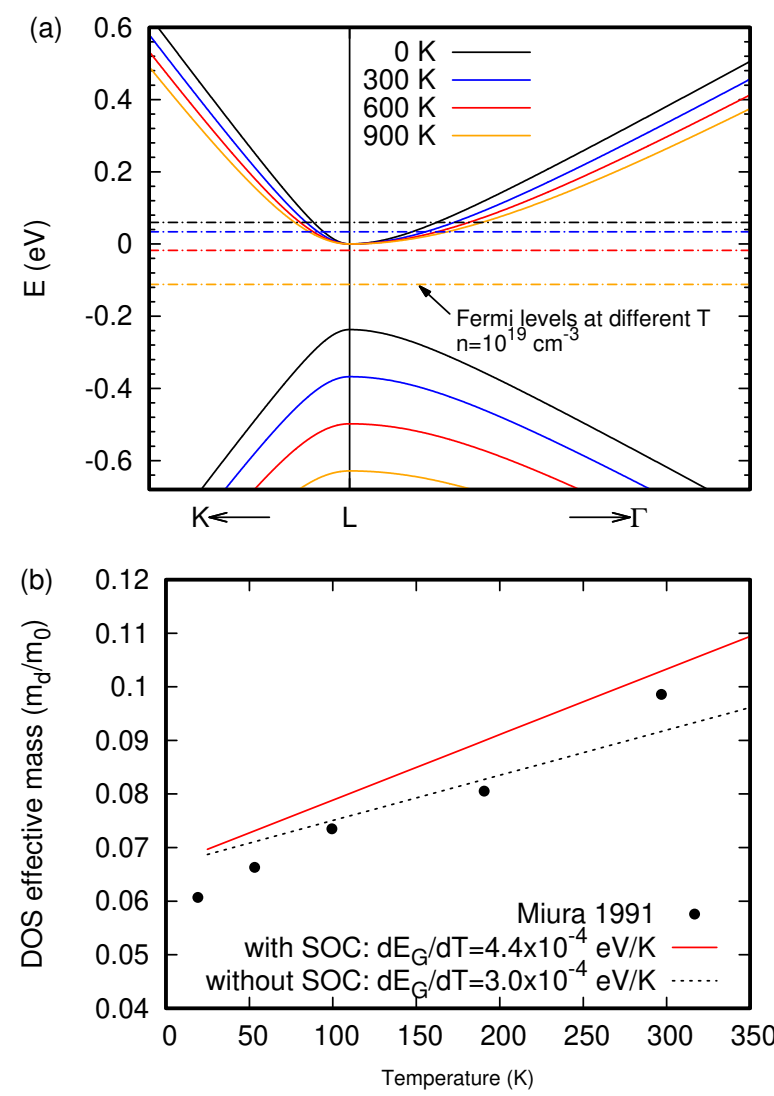

Figure 1: (a) The lowest conduction band and the highest valence band of $\mathrm{PbTe}$ near the $\mathrm{L}$ point and the Fermi level for the doping concentration of $n=10^{19} \mathrm{~cm}^{-3}$ at different temperatures calculated using $\partial E_{G} / \partial T \approx 4.4 \times 10^{-4}$ $\mathrm{eV} / \mathrm{K}$. The conduction band minima from the different temperature calculations are aligned. (b) Temperature dependence of the density-of-states (DOS) effective mass calculated with the two-band Kane model (lines) and measured experimentally (dots) [42].

Using the HSE03 hybrid functional, we obtain the band gap value of $E_{G}=0.237 \mathrm{eV}$ for PbTe, [27], which compares well to the experimental value of $0.19 \mathrm{eV}$ at $4 \mathrm{~K}$ [43]. We calculate the temperature dependence of the band gap using DFPT and the LDA excluding and including SOC. The top valence

\footnotetext{
${ }^{1}$ We note that we obtain similar values of acoustic deformation potentials using the LDA excluding SOC and the HSE03 functional including SOC [27].
} 
and bottom conduction bands at $\mathrm{L}$ of PbTe correspond to the representations $\mathrm{L}^{6+}$ and $\mathrm{L}^{6-}$, respectively [44], but that order is inverted in the LDA including SOC [27]. To account for the correct ordering of these states, we define the direct gap at $\mathrm{L}$ as $E_{G}=E_{\mathrm{L}^{6-}}-E_{\mathrm{L}^{6+}}[11]$. Using a linear fit for $E_{G}$ with respect to $T$ in the range of $200-800 \mathrm{~K}$, we compute $\partial E_{G} / \partial T \approx 3.0 \times 10^{-4} \mathrm{eV} / \mathrm{K}$ and $\partial E_{G} / \partial T \approx 4.4 \times 10^{-4} \mathrm{eV} / \mathrm{K}$ excluding and including SOC, respectively [11]. Both values compare very well to the available optical absorption measurements of $\partial E_{G} / \partial T \sim 3.0-5.1 \times 10^{-4} \mathrm{eV} / \mathrm{K}$ [8, 9, 45, 46, 47]. These results suggest that accounting for SOC or the correct order of the states near the gap does not affect the computed $\partial E_{G} / \partial T$ values very much.

The temperature dependence of the $\mathrm{CB}$ and $\mathrm{VB}$ near the $\mathrm{L}$ point is shown in Fig. 1 (a), where the CB minima from different temperature calculations are aligned. The increasing effective mass with temperature obtained in our calculations has also been observed experimentally. As shown in Fig. 1(b), the computed conduction band density-of-states (DOS) effective mass is in fairly good agreement with the measurements of Ref. [42], particularly when we use the value of $\partial E_{G} / \partial T \approx 4.4 \times 10^{-4}$ $\mathrm{eV} / \mathrm{K}$ which includes the effects of SOC. Therefore, $\partial E_{G} / \partial T \approx$ $4.4 \times 10^{-4} \mathrm{eV} / \mathrm{K}$ is used in the rest of the paper. We have verified that the conclusions of this work do not change if we use the value of $\partial E_{G} / \partial T \approx 3.0 \times 10^{-4} \mathrm{eV} / \mathrm{K}$ in our simulations.

We next show the calculated energy dependence of the inverse relaxation time associated with the four scattering mechanisms $\left(\tau_{\mathrm{ac}}^{-1}, \tau_{\mathrm{LO}}^{-1}, \tau_{\mathrm{TO}}^{-1}, \tau_{\mathrm{imp}}^{-1}\right)$ and $\tau_{\text {tot }}^{-1}$ for $T=100 \mathrm{~K}$ and $T=$ $600 \mathrm{~K}$ at the doping concentrations of $n=1 \times 10^{19} \mathrm{~cm}^{-3}$, see Fig. 2 We also plotted $(-\partial f / \partial E)$ to indicate the energy range that contributes to electronic transport and understand the relative importance of different scattering mechanisms in this range. We find that $\mathrm{LO}$ phonon scattering is the strongest scattering mechanism, while acoustic and, to a lesser extent, TO scattering become comparable only when $T$ and doping are very high. LO scattering includes long-range polar and shortrange non-polar interactions, whose relaxation times are also plotted in Fig. 22 Since the matrix elements $g_{\boldsymbol{k}}^{\boldsymbol{k}+\boldsymbol{q}}$ of these two contributions are added up, the non-polar contribution considerably modifies the energy dependence of $\tau_{\text {LO }}$ despite its relatively small magnitude. In general, ionized impurity scattering is negligible compared to electron-phonon scattering, except at low $T$ and low doping concentrations. At low temperatures $(T=100 \mathrm{~K})$, there is a dip in $\tau_{\mathrm{LO}}^{-1}$ around the Fermi level, which is a consequence of the Pauli exclusion principle for this inelastic process (see Appendix A.

The importance of accounting for the temperature dependence of the band structure (T-depBS) to compute the electronic transport properties of PbTe is illustrated in Fig. 3. The electronic mobility for $n=2.3 \times 10^{19} \mathrm{~cm}^{-3}$ as a function of temperature excluding and including the T-depBS is given by the black solid and dashed lines, respectively. Squares and circles show the mobility measurements from Refs. [40, 48]. Including the T-depBS decreases the mobility about $25 \%$ at $300 \mathrm{~K}$ and $50 \%$ at $900 \mathrm{~K}$, and gives a much better agreement with the experimental values and their temperature dependence. The
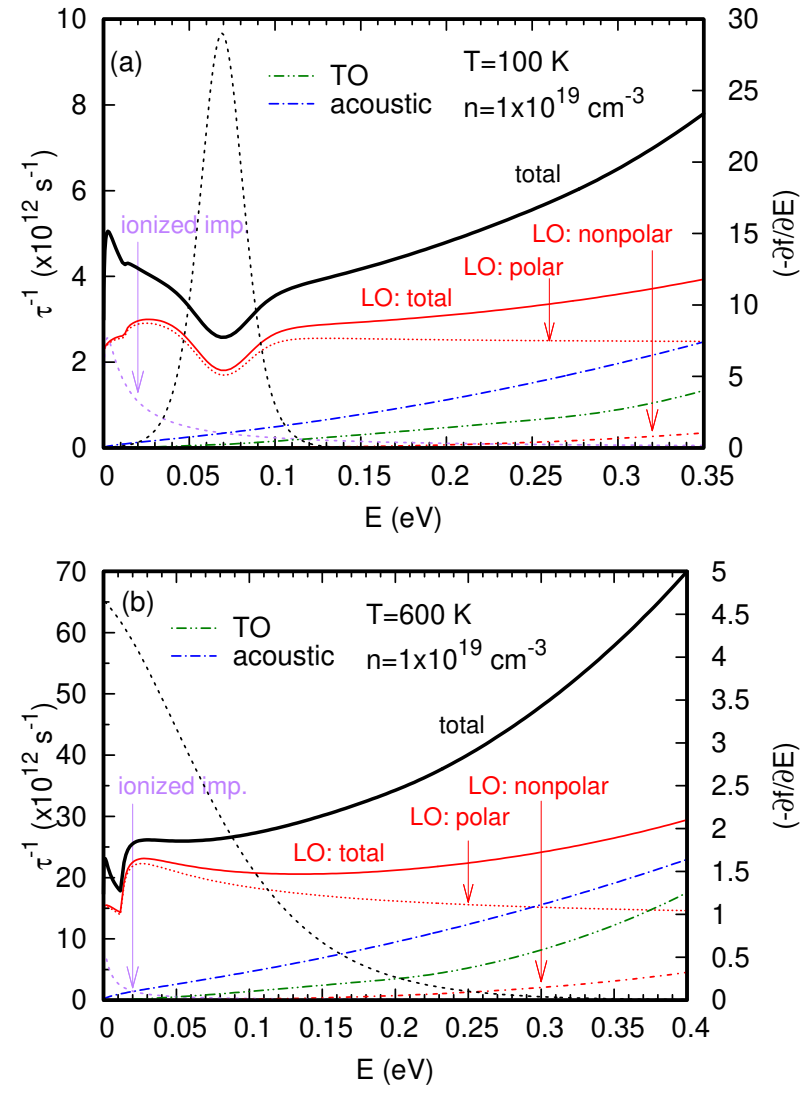

Figure 2: Calculated energy dependence of the inverse of the relaxation time $\left(\tau^{-1}\right)$ for longitudinal optical (LO), transverse optical (TO) and acoustic phonon scattering, and ionized impurity scattering in $\mathrm{PbTe}$ for the doping concentration of $n=1 \times 10^{19} \mathrm{~cm}^{-3}$ at: (a) $100 \mathrm{~K}$, and (b) $600 \mathrm{~K}$. Black dashed curves represents the derivative of the Fermi-Dirac distribution function with respect to energy, indicating the energy region contributing to electronic transport at different temperatures.

difference between our results including and excluding the TdepBS becomes larger as $T$ increases. This is because $m_{\|}^{*}$ and $m_{\perp}^{*}$ increase with $T$ due to the increased band gap, leading to a decrease of the band curvature near the band edge and an increase of the radius of constant energy surface. These effects result in smaller group velocities and a larger phase space for scattering, thus reducing the mobility.

We also calculated the individual contributions of various scattering channels to the electronic mobility of PbTe, shown by the colored lines in Fig. 3. LO phonon scattering is the dominant scattering channel limiting the mobility between $300 \mathrm{~K}$ and $900 \mathrm{~K}$, even at high doping concentrations, since screening is relatively weak due to a large dielectric constant of PbTe [23], and also does not affect the non-polar contribution. In contrast, TO phonon scattering is the weakest electron-phonon scattering mechanism even for high temperatures, since this type of scattering between the $\mathrm{CB}$ minima at $\mathrm{L}$ and the zone center TO mode is forbidden by symmetry [23]. Acoustic phonon scattering is the second strongest scattering channel, whose importance increases at high temperatures and doping concentrations.

We note that the T-depBS makes the mobility limited by individual electron-phonon scattering channels exhibit very simi- 


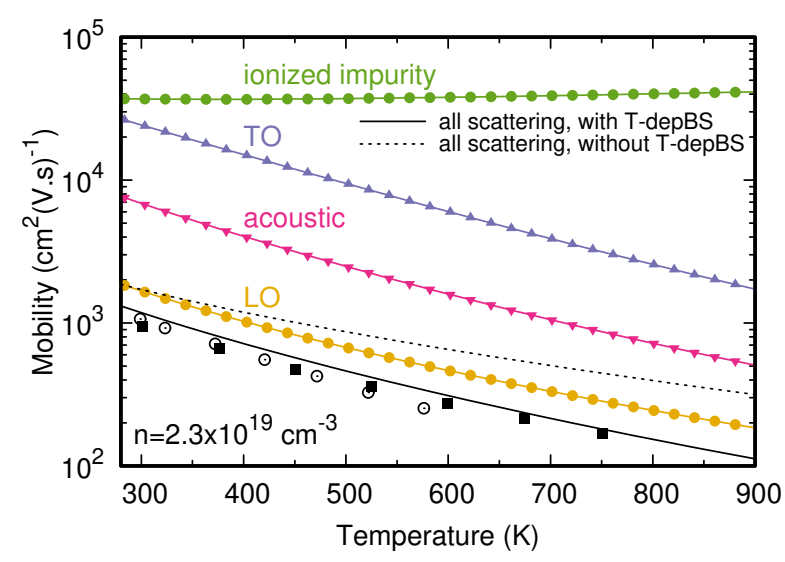

Figure 3: Electronic drift mobility versus temperature for the doping concentration of $n=2.3 \times 10^{19} \mathrm{~cm}^{-3}$. Solid black line is calculated including all scattering channels, while colored lines correspond to the contributions to the mobility from individual scattering channels. Dashed black line represents the total mobility calculated without accounting for the temperature dependence of the electronic band structure (T-depBS). Experimental data are shown in full squares (Ref. [48]) and circles (Ref. [40]).

lar $T$ dependence, as shown by the colored lines in Fig. 3 One would expect that LO phonon scattering has a characteristic $T$ dependence that is different from that of acoustic phonons due to the different energy dependence of their relaxation times $\tau$. However, the $T$ dependence of the mobility is not very sensitive to the energy dependence of $\tau$ over a large range of $T$ when the temperature dependence of the band structure is accounted for in our calculations. Instead, the $T$ dependence of the mobility comes from three sources: (1) the $T$ dependence of phonon occupations $\left(N^{0} \approx k_{B} T / \hbar \omega\right.$ for $\left.k_{B} T \gg \hbar \omega\right)$, (2) the $T$ dependence of the electronic band structure, and (3) the $T$ dependence of the Fermi level $E_{F}$. Our results suggest that it is not reliable to deduce the relative weight of different electron-phonon scattering processes only by considering the $T$ dependence of the mobility in the case of direct narrow-gap semiconductors with band gaps strongly renormalized by temperature.

The calculated temperature dependence of the electrical conductivity and the Seebeck coefficient for $n=4.8 \times 10^{18} \mathrm{~cm}^{3}$ including and excluding the T-depBS is illustrated in Fig. 4 . At high $T$, the discrepancy between the results excluding and including the T-depBS comes from two factors: (1) increasing $E_{G}$ with $T$ which reduces the hole contribution to electronic transport, and (2) increasing effective masses with $T$. To identify the role of each of these factors, we calculate the conductivity and Seebeck coefficient using the temperature-dependent gap values but keeping the effective masses constant at their $0 \mathrm{~K}$ values, shown by the red lines with empty circles in Fig. 4 The difference between the red empty and full circles shows the effect of increased hole concentration due to the increased $E_{G}$ : (1) the electrical conductivity increases, and (2) the Seebeck coefficient decreases because the energy term $\left(E_{k}-E_{F}\right)$ in Eq. 4 is negative for $S^{(\mathrm{h})}$. These effects become strong only at low doping concentrations and high $T$. On the other hand, as shown by the difference between the green full squares and the red empty circles, the temperature induced band flattening
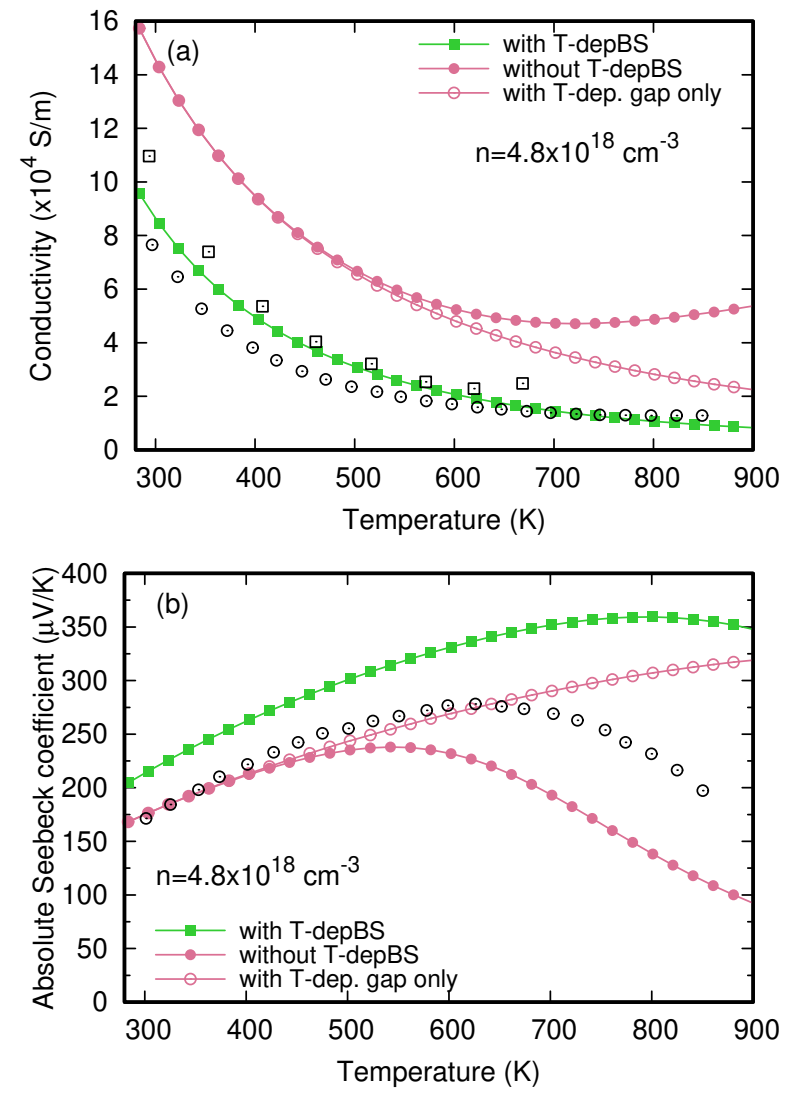

Figure 4: Calculated and experimental temperature dependence of (a) the conductivity and (b) the absolute Seebeck coefficient of n-type PbTe. Experimental data are shown in circles (Ref. [48]) and squares (Ref. [49]). T-depBS in the legend stands for the temperature dependence of the band structure, while Tdep. gap only corresponds to the temperature dependence of the band gap only.

increases the Seebeck coefficient and decreases the conductivity over the entire temperature range. We note that these effects become weaker as the doping concentration increases because the Fermi level increases and the electronic states relevant for transport are less influenced by the temperature variations of effective masses.

The comparison between the computed and measured electrical conductivity as a function of temperature for $n=4.8 \times$ $10^{18} \mathrm{~cm}^{3}$ clearly shows that it is important to include the TdepBS to obtain good agreement with experiments [48, 49] (see Fig. 4(a)). On the other hand, the Seebeck coefficient is somewhat overestimated compared to experiment when the T-depBS is accounted for (Fig. 4(b)), likely due to the fact that our first principles calculations somewhat overestimate the effective masses of PbTe (Fig. 11 b)). We also note that the calculated Seebeck coefficient for $n=4.8 \times 10^{18} \mathrm{~cm}^{-3}$ keeps increasing with temperature, and is overestimated at $T>700 \mathrm{~K}$ (see Fig. 5). In $\mathrm{PbTe}$, the valence bands at $\Sigma$ become aligned with the valence bands at $\mathrm{L}$ for $T \sim 620 \mathrm{~K}[8,11]$. This effect will also increase the hole concentration and decrease the Seebeck coefficient with respect to our present results.

The temperature dependence of the electrical conductivity, Seebeck coefficient, total and electronic contribution to the ther- 

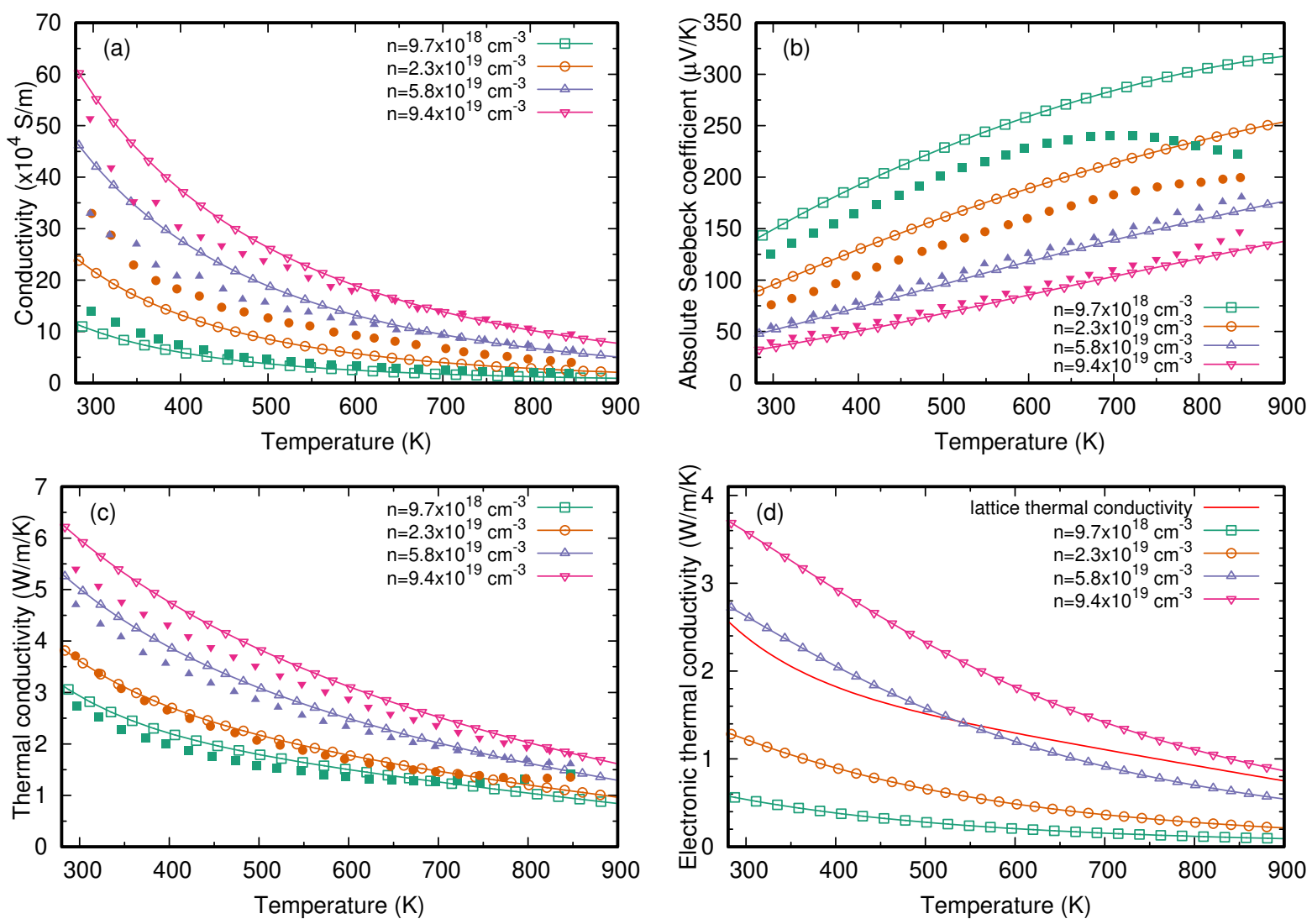

Figure 5: Calculated and experimental temperature dependence of (a) the electrical conductivity, (b) the absolute Seebeck coefficient, (c) the total thermal conductivity and (d) the electrical thermal conductivity of n-type PbTe for several doping concentrations. Experimental data are shown in full symbols (Ref. [48]).

mal conductivity for different doping concentrations $(n=9.7 \times$ $10^{18}, 2.3 \times 10^{19}, 5.8 \times 10^{19}$, and $9.4 \times 10^{19} \mathrm{~cm}^{-3}$ ) calculated including the T-depBS is plotted in Fig. 5. The lines with empty symbols represent our calculations, while the full symbols show the measurements from Ref. [48]. The lattice thermal conductivity values are taken from our previous first principles calculations [50]. For the doping concentrations above $5.8 \times 10^{19} \mathrm{~cm}^{-3}$, the electronic contribution to the thermal conductivity becomes higher than the lattice contribution. The computed TE transport properties are generally in good agreement with experiments for a wide range of doping concentrations and temperatures.

The TE transport properties of n-type PbTe as a function of the doping concentration with and without the T-depBS at $T=300 \mathrm{~K}$ are given in Appendix B We find that the calculated transport properties accounting for the T-depBS are all in very good agreement with the measurements from several experiments [40, 51, 48, 49]. Including the temperature dependence of the band structure in the first principles calculations of the thermoelectric transport properties is thus critical for accurately reproducing their dependence on the temperature and doping concentration.

We also calculate the $z T$ of n-type PbTe including and excluding the T-depBS versus $T$ and doping concentration, as shown by the color maps in Fig. 6(a) and (b). The color bar indicates the values of $z T$, while the contour lines trace the isovalues of $z T$. For a more quantitative representation, Fig. 6.c) displays the $z T$ dependence on the doping concentration at $T=300$, 600 , and $900 \mathrm{~K}$. The increasing band gap with $T$ in PbTe leads to: (1) the higher peak $z T$ value for $T$ above $650 \mathrm{~K}$, and (2) the smaller doping concentration dependence of $z T$ around its peak, which leads to the higher average $z T$ over a range of temperatures.

To understand why the T-depBS enhances the $z T$ values of n-type $\mathrm{PbTe}$, we perform a thought experiment where we vary the band gap values in the two-band Kane model independently from temperature. For each value of the gap, we also change the effective masses according to the two-band Kane model. All other parameters of our model are kept fixed. For each $T$ and band gap value, we calculate the maximal value of $z T, z T_{\max }$, by optimizing the doping concentration. Figs. 7 (a) and (b) show $z T_{\max }$ and the optimal Fermi level as a function of the band gap for $T$ ranging from $300 \mathrm{~K}$ to $900 \mathrm{~K}$. The optimal values of the gap for which $z T_{\max }$ becomes maximal are given by the circles in Fig. 7.a). For high temperatures, $z T_{\max }$ reaches a peak value at $E_{G} \approx 6.5 k_{B} T$ and then decreases with increasing band gap. For low temperatures, $z T_{\max }$ is constant for large enough gaps and does not show a clear peak. These values and trends are in agreement with the previous work of Sofo and Mahan [5].

The observed decrease of $z T_{\max }$ with decreasing band gap for $E_{g}<6 k_{B} T$ is due to the appearance of minority carriers, which strongly reduce the Seebeck coefficient and $z T$. For $E_{G}>6 k_{B} T, z T_{\max }$ can have different trends depending on the 

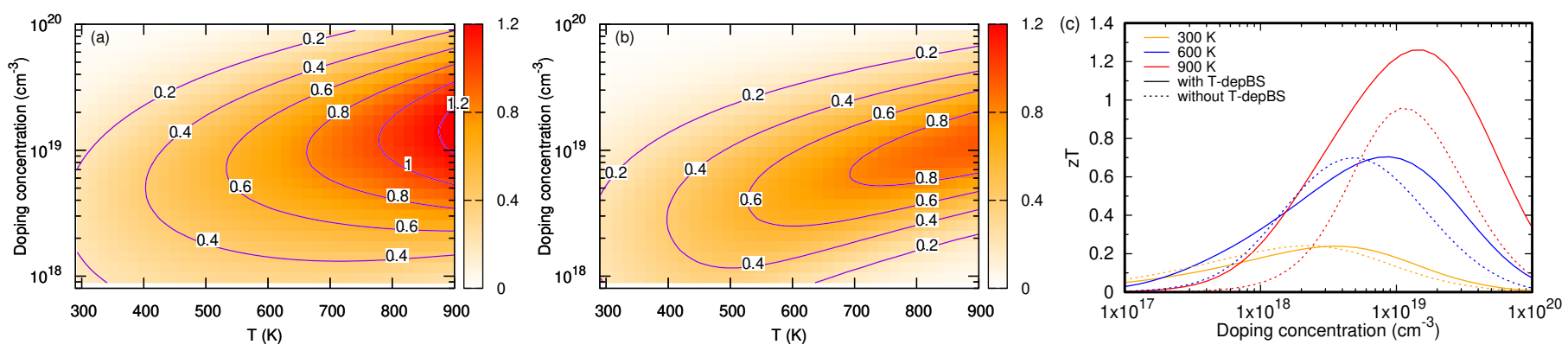

Figure 6: Color maps of the calculated figure of merit $z T$ versus temperature and doping concentration (a) including and (b) excluding the temperature dependence of the electronic band structure (T-depBS). (c) Calculated $z T$ versus doping concentration for different temperatures including and excluding T-depBS.

(a)

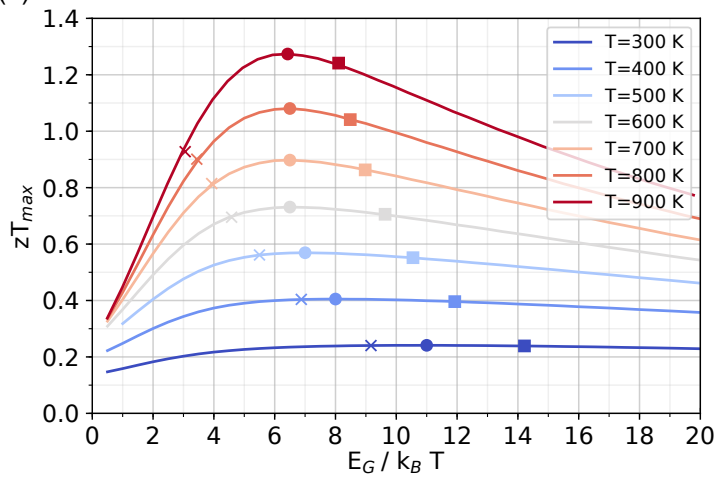

(b)

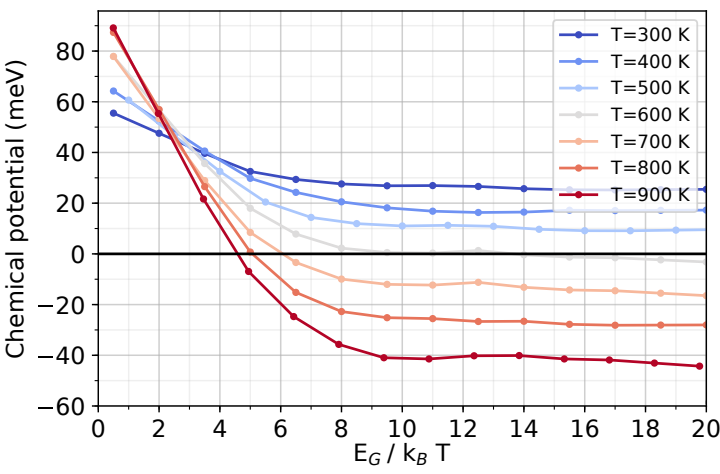

Figure 7: (a) Maximum value of $z T\left(z T_{\max }\right)$ and (b) chemical potential corresponding to $z T_{\max }$ as a function of the band gap $E_{G}$ in units of thermal energy $k_{B} T$ for different temperatures between 300 and $900 \mathrm{~K}$. $z T_{\max }$ values are obtained by adjusting the doping concentration for each $E_{G}$ and $T$. The band gap values giving maximum $z T_{\max }$ values are indicated by circles. The calculated band gap values of $\mathrm{PbTe}$ where their temperature variation is accounted for are indicated by squares, while the ground state band gap value of $E_{G}=0.237 \mathrm{eV}$ is given by crosses.

dominant scattering mechanism [5]. To gain a better insight into this, we calculate $z T_{\max }$ due to polar LO and acoustic phonon scattering, plotted in Figs. 8(a) and (b), respectively. Such $z T$ values are given on an arbitrary scale, since we are only interested in the $z T_{\max }$ trends. For acoustic scattering, $z T_{\max }$ always peaks at $E_{G}=5.5 k_{B} T$ at different temperatures. For polar LO scattering, $z T_{\max }$ rises slowly with increasing $E_{G}$. These effects (a)

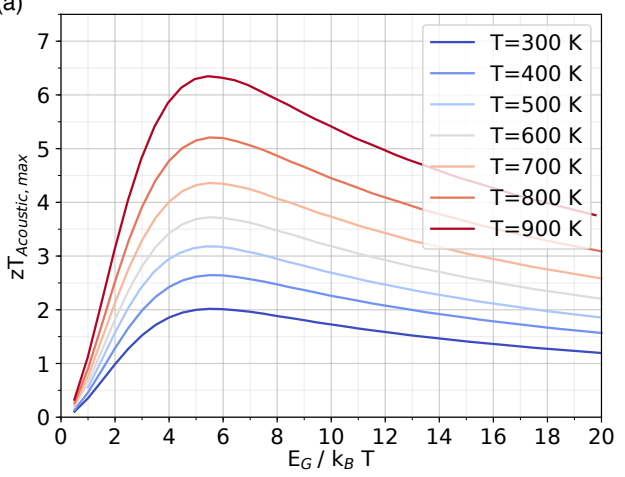

(b)

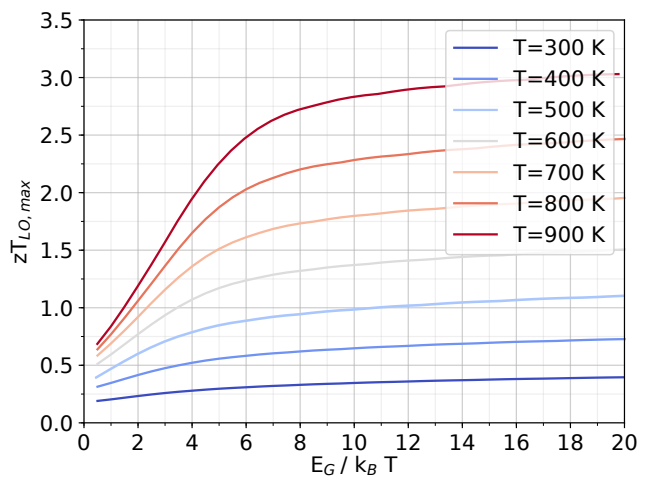

Figure 8: Maximum value of $z T\left(z T_{\max }\right)$ as function of the band gap $E_{G}$ in units of thermal energy $k_{B} T$ due to: (a) acoustic phonon scattering, and (b) polar longitudinal phonon scattering. In these plots, only the $z T_{\max }$ dependence on $E_{G}$ is relevant, while the $z T$ values on the vertical axis are given on an arbitrary scale.

are related to the dependence of the quality factor $B$, which was first introduced by Chasmar and Stratton [4], on the effective masses, which change with temperature in direct gap semiconductors [5]. In the case of PbTe, polar LO phonon scattering has a major effect at low temperatures and low doping concentrations, which is why the $z T_{\max }$ curves in Fig. 8. (a) are nearly flat around room temperature. When $T$ increases and screening becomes larger at high doping concentrations, acoustic and non-polar optical phonon scattering contributions exceed that of polar LO phonons, leading to the $z T_{\max }$ peaks in the range of 


\section{$6-10 k_{B} T$}

Finally, we analyze where the actual values of the band gap of PbTe lie on the $z T_{\max }$ curves given in Fig. 7 (a). The squares on the $z T_{\max }$ curves correspond to our calculated band gaps renormalized by temperature, while the crosses correspond to the DFT-HSE03 gap value of $E_{G}=0.237 \mathrm{eV}$ that does not change with $T$. The $z T_{\max }$ values obtained using the gap values that account for the T-depBS are very close to the $z T_{\max } \max -$ ima, especially at high temperatures, as a result of the considerable thermally induced increase of the band gan ${ }^{2}$. In contrast, if the band gap is constant (or decreasing with $T$ ), it becomes much smaller than the optimum band gap at high temperatures, leading to a substantial decrease of $z T_{\max }$. We thus conclude that the unusual increase of the band gap with temperature is an important factor for the high $z T$ of n-type $\mathrm{PbTe}$. Our results also indicate that other direct narrow-gap semiconductors with positive temperature coefficients resulting in the band gaps of $\sim$ 6-10 $k_{B} T$ may be potentially good TE materials for a relatively wide range of temperatures, similarly to $\mathrm{PbTe}$.

\section{Conclusion}

We developed the first-principles thermoelectric transport model that includes the temperature variations of the electronic band structure, and accurately describes the temperature dependence of all thermoelectric transport properties for $n$-type $\mathrm{PbTe}$ between $300 \mathrm{~K}$ and $900 \mathrm{~K}$. We also computed the optimum band gap values for which the $z T$ values of n-type PbTe would be maximized, that vary between $10 k_{B} T$ at $300 \mathrm{~K}$ and $6.5 k_{B} T$ at $900 \mathrm{~K}$. We showed that the actual band gap values in PbTe are very close to the predicted optimum values in a broad range of temperatures, which contributes largely to the good thermoelectric figure of merit of n-type PbTe in addition to its low thermal conductivity. We propose that materials with positive temperature coefficients producing the band gaps of $\sim 6-10 k_{B} T$ in a range of temperatures could be promising candidates in the search for efficient thermoelectric materials.

\section{Acknowledgement}

We thank Tchavdar Todorov for useful discussions. This work was supported by Science Foundation Ireland (SFI) under Investigators Programme No. 15/IA/3160 and Natural Science Foundation of Jiangsu Province No. BK20180456. We also acknowledge the Irish Centre for High-End Computing (ICHEC) for the provision of computational facilities.

\section{Appendix A. Inelastic scattering at low temperatures}

Fig. 2 shows a dip in $\tau_{\mathrm{LO}}^{-1}$ around the Fermi level $E_{F}$ at $T=100 \mathrm{~K}$. This is a direct consequence of the Pauli exclusion principle for this inelastic process. At low temperatures, most

\footnotetext{
${ }^{2}$ The calculated band gap values are even closer to the $z T_{\max }$ maxima at higher temperatures if we use the value of $\partial E_{G} / \partial T \approx 3.0 \times 10^{-4} \mathrm{eV} / \mathrm{K}$
}

electrons occupy the states below $E_{F}$, which are nearly completely occupied. Due to the Pauli exclusion principle, they preferentially scatter to the states above $E_{F}$, thus absorbing phonons. Since there are few thermally excited phonons at low temperatures, this scattering rate is very low. As $T$ increases, this dip disappears as electronic and phonon occupations broaden, as shown in Fig. 2(b) for $T=600 \mathrm{~K}$.

\section{Appendix B. Transport Properties at Room Temperature}

The calculated thermoelectric transport properties as a function of the doping concentration for n-type PbTe at $300 \mathrm{~K}$ including and excluding the temperature dependence of the electronic band structure (T-depBS) are illustrated in Fig. B.9. The colored lines represent our calculations while the symbols show the measurements from various experiments [40, 51, 48, 49]. The calculated transport properties that account for the T-depBS are all in very good agreement with the measurements. Including the T-depBS yields lower mobility and conductivity, and higher absolute Seebeck coefficient.

\section{References}

[1] G. J. Snyder, E. S. Toberer, Complex thermoelectric materials, Nat. Mater. 7 (2) (2008) 105-114. doi:10.1038/nmat2090

[2] F. Rosi, B. Abeles, R. Jensen, Materials for thermoelectric refrigeration, J. Phys. Chem. Solids 10 (2) (1959) $191-200$. doi:https://doi. org/10.1016/0022-3697(59)90074-5

[3] E. O. Kane, Band structure of indium antimonide, J. Phys. Chem. Solids 1 (4) (1957) 249 - 261. doi:https://doi.org/10.1016/ 0022-3697 (57) 90013-6

[4] R. P. Chasmar, R. Stratton, The thermoelectric figure of merit and its relation to thermoelectric generators $\dagger$, Journal of Electronics and Control 7 (1) (1959) 52-72. doi:10.1080/00207215908937186

[5] J. O. Sofo, G. D. Mahan, Optimum band gap of a thermoelectric material, Phys. Rev. B 49 (7) (1994) 4565-4570. doi:10.1103/physrevb.49. 4565

[6] G. D. Mahan, Figure of merit for thermoelectrics, J. Appl. Phys. 65 (4) (1989) 1578-1583. doi:10.1063/1.342976

[7] M. Cardona, M. L. W. Thewalt, Isotope effects on the optical spectra of semiconductors, Rev. Mod. Phys. 77 (2005) 1173-1224. doi:10.1103/ RevModPhys.77.1173

[8] Z. M. Gibbs, H. Kim, H. Wang, R. L. White, F. Drymiotis, M. Kaviany, G. Jeffrey Snyder, Temperature dependent band gap in $\mathrm{pbx}(\mathrm{x}=\mathrm{s}, \mathrm{se}, \mathrm{te})$, Appl. Phys. Lett. 103 (26) (2013) 262109. doi:10.1063/1.4858195

[9] A. F. Gibson, Single contact lead telluride photocells, Proc. Phys. Soc. London, Sect. B 65 (3) (1952) 196.

[10] R. Yu, E. S. Bozin, M. Abeykoon, B. Sangiorgio, N. A. Spaldin, C. D. Malliakas, M. G. Kanatzidis, S. J. L. Billinge, Emphanitic anharmonicity in pbse at high temperature and anomalous electronic properties in the $\mathrm{Pb} q(q=\mathrm{S}, \mathrm{Se}, \mathrm{Te})$ system, Phys. Rev. B 98 (2018) 144108. doi:10. 1103/PhysRevB.98.144108

[11] J. D. Querales-Flores, J. Cao, S. Fahy, I. Savić, Temperature effects on the electronic band structure of pbte from first principles, Phys. Rev. Materials 3 (2019) 055405. doi:10.1103/PhysRevMaterials.3.055405

[12] T. J. Scheidemantel, C. Ambrosch-Draxl, T. Thonhauser, J. V. Badding, J. O. Sofo, Transport coefficients from first-principles calculations Phys. Rev. B 68 (2003) 125210. doi:10.1103/PhysRevB.68.125210 URL https://link.aps.org/doi/10.1103/PhysRevB.68. 125210

[13] D. J. Singh, Doping-dependent thermopower of PbTe from Boltzmann transport calculations Phys. Rev. B 81 (19) (2010) 195217. doi:10. 1103/PhysRevB.81.195217

URL/http://link.aps.org/doi/10.1103/PhysRevB.81.195217 

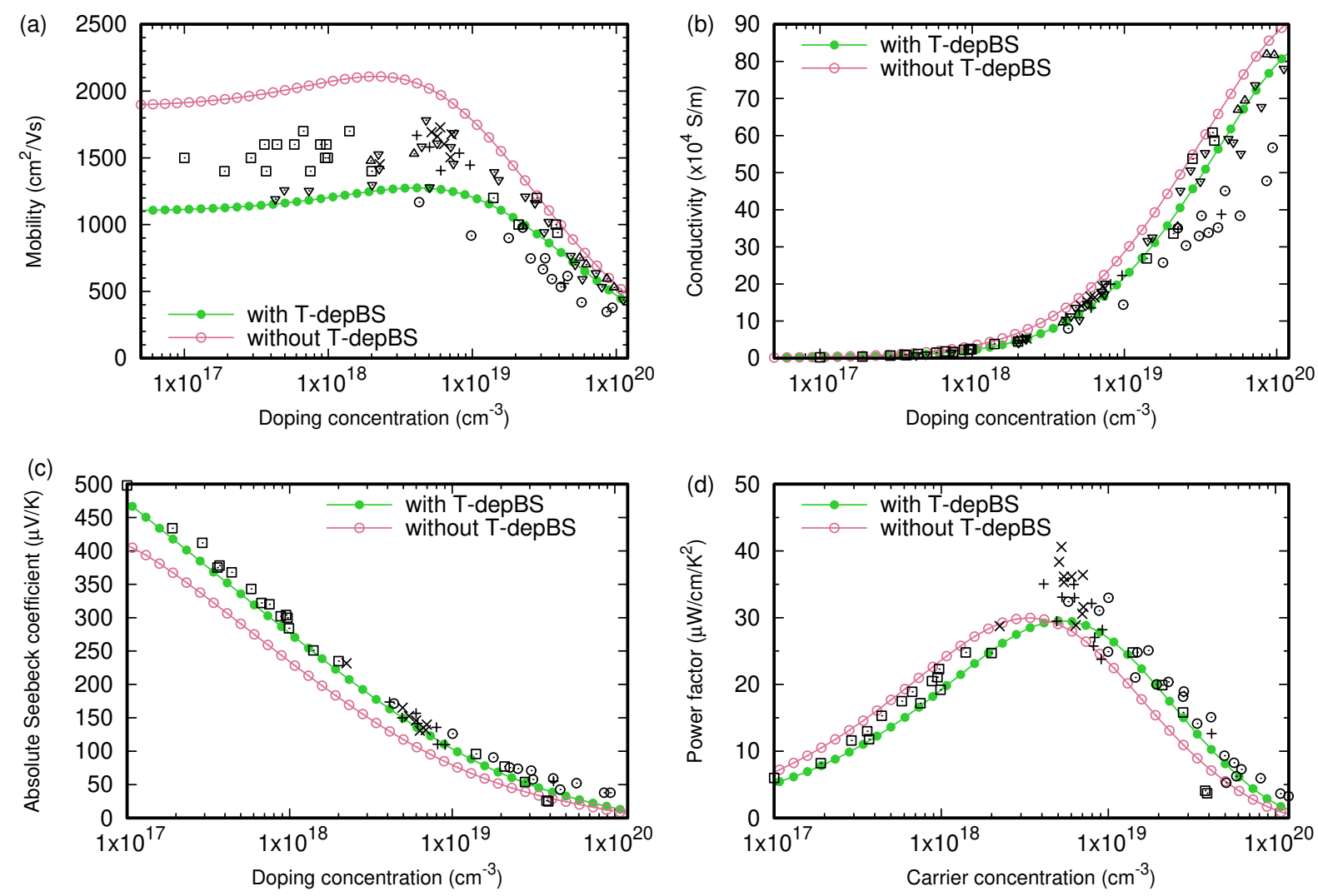

Figure B.9: Calculated and experimental dependence of (a) the mobility, (b) the conductivity, (c) the absolute Seebeck coefficient and (d) the power factor on the doping concentration at $T=300 \mathrm{~K}$. Experimental data are shown in squares (Ref. [51]), circles (Ref. [48]), triangles up and down (Ref. [40]), and crosses (Ref. [49]).

[14] T. Sohier, M. Calandra, C.-H. Park, N. Bonini, N. Marzari, F. Mauri, Phonon-limited resistivity of graphene by first-principles calculations: Electron-phonon interactions, strain-induced gauge field, and boltzmann equation, Phys. Rev. B 90 (2014) 125414. doi:10.1103/PhysRevB. 90.125414

[15] T. Gunst, T. Markussen, K. Stokbro, M. Brandbyge, First-principles method for electron-phonon coupling and electron mobility: Applications to two-dimensional materials, Phys. Rev. B 93 (3) (2016) 035414 doi:10.1103/PhysRevB.93.035414

[16] M. Fiorentini, N. Bonini, Thermoelectric coefficients of $n$-doped silicon from first principles via the solution of the boltzmann transport equation, Phys. Rev. B 94 (2016) 085204. doi:10.1103/PhysRevB.94.085204

[17] Q. Song, T.-H. Liu, J. Zhou, Z. Ding, G. Chen, Ab initio study of electron mean free paths and thermoelectric properties of lead telluride, Mater. Today Phys. 2 (2017) 69 - 77. doi:https://doi.org/10.1016/j. mtphys.2017.09.002

[18] J. Ma, Y. Chen, W. Li, Intrinsic phonon-limited charge carrier mobilities in thermoelectric snse, Phys. Rev. B 97 (2018) 205207. doi:10.1103/ PhysRevB.97.205207

[19] S. Poncé, E. R. Margine, F. Giustino, Towards predictive many-body calculations of phonon-limited carrier mobilities in semiconductors, Phys. Rev. B 97 (2018) 121201. doi:10.1103/PhysRevB.97.121201

[20] J.-J. Zhou, O. Hellman, M. Bernardi, Electron-phonon scattering in the presence of soft modes and electron mobility in srtio 3 perovskite from first principles, Phys. Rev. Lett. 121 (2018) 226603. doi:10.1103/ PhysRevLett.121.226603

[21] D. I. Bilc, S. D. Mahanti, M. G. Kanatzidis, Electronic transport properties of PbTe and agpb ${ }_{m}$ sbte $_{2+m}$ systems, Phys. Rev. B 74 (2006) 125202. doi:10.1103/PhysRevB.74.125202

[22] S. Ahmad, S. D. Mahanti, Energy and temperature dependence of relaxation time and wiedemann-franz law on pbte, Phys. Rev. B 81 (2010) 165203. doi:10.1103/PhysRevB.81.165203
[23] J. Cao, J. D. Querales-Flores, A. R. Murphy, S. Fahy, I. Savić, Dominant electron-phonon scattering mechanisms in $n$-type pbte from first principles, Phys. Rev. B 98 (2018) 205202. doi:10.1103/PhysRevB.98. 205202

[24] G. Kresse, J. Furthmüller, Efficiency of ab-initio total energy calculations for metals and semiconductors using a plane-wave basis set, Comput. Mater. Sci. 6 (1) (1996) 15 - 50. doi:https://doi.org/10.1016/ 0927-0256 (96) 00008-0

[25] J. Heyd, G. E. Scuseria, M. Ernzerhof, Hybrid functionals based on a screened coulomb potential, J. Chem. Phys. 118 (18) (2003) 8207-8215. doi:10.1063/1.1564060

[26] J. Heyd, G. E. Scuseria, Efficient hybrid density functional calculations in solids: Assessment of the heyd-scuseria-ernzerhof screened coulomb hybrid functional, J. Chem. Phys. 121 (3) (2004) 1187-1192. doi:10. 1063/1.1760074

[27] A. R. Murphy, F. Murphy-Armando, S. Fahy, I. Savić, Acoustic deformation potentials of $n$-type pbte from first principles, Phys. Rev. B 98 (2018) 085201. doi:10.1103/PhysRevB.98.085201

[28] G. Kresse, D. Joubert, From ultrasoft pseudopotentials to the projector augmented-wave method, Phys. Rev. B 59 (1999) 1758-1775. doi:10. 1103/PhysRevB.59.1758

[29] Y. W. Tsang, M. L. Cohen, Calculation of the temperature dependence of the energy gaps in pbte and snte, Phys. Rev. B 3 (1971) 1254-1261. doi:10.1103/PhysRevB.3.1254

[30] M. Schlüter, G. Martinez, M. L. Cohen, Pressure and temperature dependence of electronic energy levels in pbse and pbte, Phys. Rev. B 12 (1975) 650-658. doi:10.1103/PhysRevB.12.650

[31] P. B. Allen, V. Heine, Theory of the temperature dependence of electronic band structures, J. Phys. C Solid State 9 (12) (1976) 2305.

[32] P. B. Allen, M. Cardona, Theory of the temperature dependence of the direct gap of germanium, Phys. Rev. B 23 (1981) 1495-1505. doi:10. 1103/PhysRevB.23.1495 
[33] P. B. Allen, M. Cardona, Temperature dependence of the direct gap of si and ge, Phys. Rev. B 27 (1983) 4760-4769. doi:10.1103/PhysRevB. 27.4760

[34] X. Gonze, B. Amadon, P.-M. Anglade, J.-M. Beuken, F. Bottin, P. Boulanger, F. Bruneval, D. Caliste, R. Caracas, M. Côté, T. Deutsch, L. Genovese, P. Ghosez, M. Giantomassi, S. Goedecker, D. Hamann, P. Hermet, F. Jollet, G. Jomard, S. Leroux, M. Mancini, S. Mazevet, M. Oliveira, G. Onida, Y. Pouillon, T. Rangel, G.-M. Rignanese, D. Sangalli, R. Shaltaf, M. Torrent, M. Verstraete, G. Zerah, J. Zwanziger, Abinit: First-principles approach to material and nanosystem properties, Comput. Phys. Commun. 180 (12) (2009) 2582 - 2615. doi:https : //doi.org/10.1016/j.cpc.2009.07.007

[35] X. Gonze, F. Jollet, F. Abreu Araujo, D. Adams, B. Amadon, T. Applencourt, C. Audouze, J.-M. Beuken, J. Bieder, A. Bokhanchuk, E. Bousquet, F. Bruneval, D. Caliste, M. Côté, F. Dahm, F. Da Pieve, M. Delaveau, M. Di Gennaro, B. Dorado, C. Espejo, G. Geneste, L. Genovese, A. Gerossier, M. Giantomassi, Y. Gillet, D. Hamann, L. He, G. Jomard, J. Laflamme Janssen, S. Le Roux, A. Levitt, A. Lherbier, F. Liu, I. Lukačević, A. Martin, C. Martins, M. Oliveira, S. Poncé, Y. Pouillon, T. Rangel, G.-M. Rignanese, A. Romero, B. Rousseau, O. Rubel, A. Shukri, M. Stankovski, M. Torrent, M. Van Setten, B. Van Troeye, M. Verstraete, D. Waroquiers, J. Wiktor, B. Xu, A. Zhou, J. Zwanziger, Recent developments in the ABINIT software package, Comput. Phys. Commun. 205 (2016) 106-131. doi:10.1016/j.cpc.2016.04.003

[36] D. M. Ceperley, B. J. Alder, Ground state of the electron gas by a stochastic method, Phys. Rev. Lett. 45 (1980) 566-569. doi:10.1103/ PhysRevLett.45.566

[37] J. P. Perdew, A. Zunger, Self-interaction correction to density-functional approximations for many-electron systems, Phys. Rev. B 23 (1981) 50485079. doi:10.1103/PhysRevB.23.5048

[38] C. Hartwigsen, S. Goedecker, J. Hutter, Relativistic separable dual-space Gaussian pseudopotentials from H to Rn, Phys. Rev. B 58 (7) (1998) 3641-3662. doi:10.1103/PhysRevB.58.3641

[39] M. S. Dresselhaus, G. Dresselhaus, A. Jorio, Group Theory: Application to the Physics of Condensed Matter, 1st Edition, Springer-Verlag Berlin Heidelberg, 2008.

[40] Y. I. Ravich, B. A. Efimova, V. I. Tamarchenko, Scattering of Current Carriers and Transport Phenomena in Lead Chalcogenides II. Experiment, Phys. Status Solidi (b) 43 (2) (1971) 453-469. doi:10.1002/pssb. 2220430202

[41] D. M. Zayachuk, The dominant mechanisms of charge-carrier scattering in lead telluride, Semiconductors 31 (2) (1997) 173-176. doi:10.1134/ 1.1187322

[42] H. Yokoi, S. Takeyama, N. Miura, G. Bauer, Anomalous temperature dependence of the effective mass in n-type pbte, Phys. Rev. B 44 (1991) 6519-6522. doi:10.1103/PhysRevB.44.6519

[43] R. Dalven, Electronic Structure of PbS, PbSe, and PbTe, Vol. 28 of Solid State Physics, Academic Press, 1974. doi:10.1016/S0081-1947(08) 60203-9

[44] S. E. Kohn, P. Y. Yu, Y. Petroff, Y. R. Shen, Y. Tsang, M. L. Cohen, Electronic band structure and optical properties of pbte, pbse, and pbs, Phys. Rev. B 8 (1973) 1477-1488. doi:10.1103/PhysRevB.8.1477

[45] R. N. Tauber, A. A. Machonis, I. B. Cadoff, Thermal and optical energy gaps in pbte, J. Appl. Phys. 37 (13) (1966) 4855-4860. doi:10.1063/ 1.1708150

[46] V. A. Saakyan, E. D. Devyatkova, I. A. Smirnov, Sov. Phys. Solid State 7 (1966) 2541-2542.

[47] M. Baleva, T. Georgiev, G. Lashkarev, On the temperature dependence of the energy gap in pbse and pbte, J. Phys. Condens. Matter 2 (13) (1990) 2935.

[48] Y. Pei, Z. M. Gibbs, A. Gloskovskii, B. Balke, W. G. Zeier, G. J. Snyder, Optimum carrier concentration in n-type pbte thermoelectrics, Adv. Energy Mater. 4 (13) (2014) 1400486. doi:10.1002/aenm. 201400486

[49] C. J. Vineis, T. C. Harman, S. D. Calawa, M. P. Walsh, R. E. Reeder, R. Singh, A. Shakouri, Carrier concentration and temperature dependence of the electronic transport properties of epitaxial pbte and pbte/pbse nanodot superlattices, Phys. Rev. B 77 (2008) 235202. doi:10.1103/ PhysRevB.77.235202

[50] R. M. Murphy, E. D. Murray, S. Fahy, I. Savić, Broadband phonon scattering in pbte-based materials driven near ferroelectric phase transition by strain or alloying, Phys. Rev. B 93 (2016) 104304. doi:
10.1103/PhysRevB.93.104304

[51] L. D. Hicks, T. C. Harman, X. Sun, M. S. Dresselhaus, Experimental study of the effect of quantum-well structures on the thermoelectric figure of merit, Phys. Rev. B 53 (1996) R10493-R10496. doi: 10.1103/PhysRevB.53.R10493 